\title{
SUMMATION OF MULTIPLE FOURIER SERIES BY SPHERICAL MEANS*
}

BY

\author{
SALOMON BOCHNER
}

PART I. AN OUTLINE OF THE RESUlts

Let $x=\left(x_{1}, \cdots, x_{k}\right)$ be a point in the $k$-dimensional Euclidean space and $f(x)=f\left(x_{1}, \cdots, x_{k}\right)$ a function of the Lebesgue class $L$ having the period $2 \pi$ in each variable; let

$$
f(x) \sim \sum a_{n_{1} \cdots n_{k}} e^{i\left(n_{1} x_{1}+\cdots+n_{k} x k\right)}
$$

be its Fourier series,

$$
a_{n_{1} \cdots n_{k}}=\frac{1}{(2 \pi)^{k}} \int_{-\pi}^{\pi} \cdots \int_{-\pi}^{\pi} f(x) e^{-i\left(n_{1} x_{1}+\cdots+n_{k} x_{k}\right)} d x .
$$

We shall consider criteria for (convergence and) summability not of the rectangular partial sums

$$
\sum_{-N_{1}}^{N_{1}} \cdots \sum_{-N_{k}}^{N_{k}} a_{n_{1} \cdots n_{k}} e^{i\left(n_{1} x_{1}+\cdots+n_{k} x_{k}\right)}
$$

but of the spherical partial sums

$$
S_{R}(x)=\sum_{\nu \leqq R} a_{n_{1} \cdots n_{k}} e^{i\left(n_{1} x_{1}+\cdots+n_{k} x_{k}\right)} ; \quad \nu^{2}=n_{1}{ }^{2}+\cdots+n_{k}{ }^{2} .
$$

This means that we shall consider the series (1) as the simple series

$$
\sum_{j=0}^{\infty} \sum_{\nu=R} a_{n_{1} \cdots n_{k}} e^{i\left(n_{1} x_{1}+\cdots+n_{k} x_{k}\right)},
$$

where $R_{j}^{2}$ is the sequence of all integers that can be represented as a sum of $k$ squares of integers. (For $k \geqq 4$ the sequence consists of all non-negative integers.) In summing series (5) we shall restrict ourselves to Toeplitz matrices $\left\{a_{p q}\right\}$ in which $a_{p q}=\phi(p / q)$. Thus we shall consider, and take the limit of, as $R \rightarrow \infty$, the "partial sums"

$$
S_{R}^{\phi}(x) \sim \sum \phi\left(\frac{\nu}{R}\right) a_{n_{1} \cdots n_{k}} e^{i\left(n_{1} x_{1}+\cdots+n_{k} x_{k}\right)}
$$

* Presented to the Society, April 20, 1935; received by the editors October 11, 1935.

A brief summary of the results was published in the Proceedings of the National Academy of Sciences, vol. 21 (1935), pp. 353-355. 
(the sum to be extended over all combinations of integers $n_{1}, \cdots, n_{k}$ ), where $\phi(t)$ is a fixed function in $0 \leqq t<\infty$ for which $\phi(0)=1$. We note that we shall allow the index $R$ to range over all values $0 \leqq R<\infty$ and not only over the sequence $\left\{R_{j}\right\}$.

Interesting cases arise when $\phi(t)$ is one of the following functions:

$$
K_{\delta}(t)=\left\{\begin{array}{cl}
e^{-t} & \text { Poisson-Abel summation, } \\
e^{-t^{2}}, & \text { Gauss-Sommerfeld summation, } \\
\left(1-t^{2}\right)^{\delta} & \text { for } 0 \leqq t<1, \\
0 & \text { for } 1 \leqq t,
\end{array}\right.
$$

the latter being the important case of Riesz summation of order $\delta .^{*}$

The spherical partial sums (6) have a great advantage over the rectangular partial sums (3). This will be evident from the formula

$$
S_{R}^{\phi}(x)=R \int_{0}^{\infty} f_{x}(t) H_{\phi}(t R) d t
$$

which we are going to explain presently (its validity will be discussed later on). We shall use throughout the abbreviations

$$
\begin{aligned}
& l=\frac{k-2}{2}, \\
& \alpha=\frac{k-1}{2}, \\
& c=t R,
\end{aligned}
$$

and by $J_{l}(u)$ we shall denote the Bessel function of order $l$. The kernel $H_{\phi}(c)$ depends only on $\phi(t)$ and not on $f(x)$ :

$$
\begin{aligned}
H_{\phi}(c) & =\frac{1}{c} \int_{0}^{\infty} \phi\left(\frac{u}{c}\right) u^{l+1} J_{l}(u) d u \\
& =c^{l+1} \int_{0}^{\infty} \phi(u) u^{l+1} J_{l}(u c) d u .
\end{aligned}
$$

Thus

$$
\begin{array}{ll}
H_{\phi}(c)=\frac{2^{l+1} \Gamma\left(l+\frac{3}{2}\right)}{\pi^{1 / 2}} \cdot \frac{c^{2 l+1}}{\left(1+c^{2}\right)^{l+3 / 2}} & \text { if } \phi(t)=e^{-t}, \\
H_{\phi}(c)=\frac{c^{2 l+1}}{2^{l+1}} \cdot e^{-c^{2} / 4} & \text { if } \phi(t)=e^{-t^{2}},
\end{array}
$$

\footnotetext{
* It is more convenient to consider the Riesz factor $\left(1-t^{2}\right)^{\delta}$ than $(1-t)^{\delta}$; see formula (18).
} 
(18)
$H_{\phi}(c) \equiv H_{\delta}(c)=2^{\delta} \Gamma(\delta+1) \cdot \frac{J_{\delta+l+1}(c)}{c^{\delta-l}}$
if $\phi(t)=K_{8}(t),{ }^{*}$

and, in particular,

$$
H_{\alpha}(c)=2^{\alpha} \Gamma(\alpha+1) \frac{J_{k-1 / 2}(c)}{c^{1 / 2}} \quad \text { if } \phi(t)=K_{\alpha}(t) .
$$

The factor $f_{x}(t)$ in the integral (10) is determined by the given function $f(x)=f\left(x_{1}, \cdots, x_{k}\right)$. Unlike $f(x)$ it is a function of one variable only; its argument $t$ ranges over the interval $0 \leqq t<\infty$. Its value is, apart from a factor depending only on $k$, the $(k-1)$-dimensional spherical mean of the function $f(x)$ over a sphere whose radius is $t$ and whose center is at the point $x$. If $\sigma$ denotes the unit sphere $\xi_{1}^{2}+\cdots+\xi_{k}^{2}=1$ and $d \sigma_{\xi}$ its $(k-1)$-dimensional volume element, then

$$
f_{x}(t)=\frac{1}{(2 \pi)^{k / 2}} \int_{\sigma} f\left(x_{1}+t \xi_{1}, \cdots, x_{k}+t \xi_{k}\right) d \sigma_{\xi} .
$$

For $k=1$ the integral over the unit sphere is to be replaced by $f(x+t)+f(x-t)$. We shall require the explicit value of $f_{x}(t)$ only for

$$
f(x)=e^{i\left(n_{1} x_{1}+\cdots+n_{k} x_{k}\right)} .
$$

In this case we have

$$
f_{x}(t)=f_{0}(t) f(x)
$$

Moreover

$$
f_{0}(t)=\frac{J_{l}(\nu t)}{(\nu t)^{l}} .
$$

In fact, it is easily seen that

$$
f_{0}(t)=\frac{\omega_{k-1}}{(2 \pi)^{k / 2}} \int_{0}^{\pi} e^{i v t \cos \theta}(\sin \theta)^{k-2} d \theta,
$$

where $\omega_{k}$ is the $(k-1)$-dimensional volume of the unit sphere $\xi_{1}^{2}+\cdots+\xi_{k}^{2}$ $=1$. Now apply formula (6) in Watson, loc. cit., p. 48 .

As soon as formula (10) holds, what decides the convergence of the partial sums (6) at a given point $x$ are only the spherical averages of the functions $f(x)$ around the one point $x$ and nothing else. It must be pointed out that formula (10), as it stands, involves the function $f_{x}(t)$ in its entire infinite in-

* See G. N. Watson, Bessel Functions, p. 386, formula (6); p. 394, formula (4); p. 373, formula (1). 
terval $0 \leqq t<\infty$ and not only in some such finite $t$-interval that might cover a fundamental domain of periodicity of $f(x)$. However, if $\phi(t)$ has a sufficient number of derivatives which are sufficiently small at infinity or, in case $\phi(t)=K_{\delta}(t)$, if $\delta>\alpha$, then the limit behaviour of $S_{R} \phi(x)$, that is, its convergence or non-convergence as $R \rightarrow \infty$, depends only on the values of $f_{x}(t)$ in any interval $0 \leqq t<t_{0}$, no matter how small; in particular the partial sum $S_{R^{\phi}}(x)$ converges to 0 if the function $f(x)$ vanishes in some neighborhood of the point $x$ (Theorem VI).

This localization property is the more remarkable since it is highly characteristic of our spherical method of summation. In the case of "rectangular" or even "square" partial sums, that is, in case we consider

$$
\sigma_{R}^{\phi}(x) \sim \sum \phi\left(\frac{\left|n_{1}\right|}{R}\right) \cdots \phi\left(\frac{\left|n_{k}\right|}{R}\right) a_{n_{1} \cdots n_{k}} e^{i\left(n_{1} x_{1}+\cdots+n_{k} x_{k}\right)},
$$

it fails to hold for such a smooth summation function as $\phi(t)=e^{-t}$ is (Theorem VIII).

As far as our Theorems I and V are concerned, a function $f(x)$ has a "limit" at a point $x$ as soon as $f_{x}(t)$ is continuous for $t=0$. In the case of rectangular summation the corresponding condition requires continuity in "octants," that is, the existence of the $2^{k}$ limits $f\left(x_{1} \pm 0, \cdots, x_{k} \pm 0\right)$. This condition is rather more restrictive. The nearest approach to our limit "in spherical average" is the one occurring in C. N. Moore's criterion for summation of "restricted" rectangular partial sums. The condition also requires continuity in "octants," but the octants may be bounded by very general curves instead of by straight lines.*

In order to safeguard the localization property under Riesz summation it is not only sufficient but also necessary to require that $\delta>\alpha$. That the localization property no longer holds for $\delta<\alpha$ suggests itself readily and can be proved rather easily. The situation is less obvious in the case of the critical exponent $\delta=\alpha$ itself. For $k=1$, in which case $\alpha=0$, it is a fundamental result of Riemann that the localization property holds for $\delta=\alpha$. But, as we shall see, the contrary is true for every $k \geqq 2$. If $k \geqq 2$ then there exists a function $f(x)$ of Lebesgue class $L$ vanishing in the neighborhood of the point $0=(0, \cdots, 0)$ for which the partial sums $S_{R^{\alpha}}{ }^{\alpha}(0)$ are not bounded as $R \rightarrow \infty$ (Theorem VII).

However, the localization property does hold also for the critical ex-

* C. N. Moore, On the summability of the double Fourier series of discontinuous functions, Mathematische Annalen, vol. 74 (1913), pp. 555-572; esp. p. 567, Theorem III. 
ponent $\delta=\alpha$ if instead of Fourier series we consider Fourier integrals. Let $f(x)$ be a (non-periodic) function belonging to class $L$ over the whole space. Replacing (1), (2), (6) by

$$
\begin{aligned}
& f(x) \sim \int \cdots \int_{-\infty}^{\infty} a\left(n_{1}, \cdots, n_{k}\right) e^{i\left(n_{1} x_{1}+\cdots+n_{k} x_{k}\right)} d n_{1} \cdots d n_{k} \\
& a\left(n_{1}, \cdots, n_{k}\right)=\frac{1}{(2 \pi)^{k}} \int \cdots \int_{-\infty}^{\infty} f(x) e^{-i\left(n_{1} x_{1}+\cdots+n_{k} x_{k}\right)} d x_{1} \cdots d x_{k} \\
& S_{R}^{\phi}(x) \sim \int \cdots \int_{-\infty}^{\infty} \phi\left(\frac{\nu}{R}\right) a\left(n_{1}, \cdots, n_{k}\right) e^{i\left(n_{1} x_{1}+\cdots+n_{k} x_{k}\right)} d n_{1} \cdots d n_{k}
\end{aligned}
$$

the relations (10)-(20) remain valid without any change whatsoever. Many results are analogous for the two groups of expansions, and in fact the Fourier integrals are easier than the Fourier series. A study of spherical summation in case of Fourier integrals was made by the author some time ago, ${ }^{*}$ and in the present paper we shall give a more detailed account.

The spherical average $f_{x}(t)$ and the critical exponent (12) for Riesz summation of multiple Fourier series are not new. They have been known with reference to expansions in ultra-spherical functions.

The elementary exponentials

$$
u(x)=e^{i\left(n_{1} x_{1}+\cdots+n_{k} x_{k}\right)}
$$

(ail $n_{1}, \cdots, n_{k}$ integers) are a complete set of regular solutions of the characteristic value problem

$$
\Delta u(x)=-\lambda u(x),
$$

if this equation is being considered on the (closed) torus

$$
0 \leqq x_{1}<2 \pi, \cdots, 0 \leqq x_{k}<2 \pi,
$$

and $\Delta u(x)$ is the Laplace operator with respect to the Euclidean metric on the torus, namely,

$$
\frac{\partial^{2} u}{\partial x_{1}^{2}}+\cdots+\frac{\partial^{2} u}{\partial x_{k}^{2}}
$$

Since $\lambda=n_{1}^{2}+\cdots+n_{k}^{2}$, our way of writing series (1) in the form (5) satisfies the very natural principle of ordering the terms in series (1) according to the magnitude of the characteristic values $\lambda$. Now the characteristic

* See S. Bochner, Ein Konvergenzsatz fïr mehrvariablige Fouriersche Integrale, Mathematische Zeitschrift, vol. 34 (1931), pp. 440-447; analogous ideas are found also in earlier work of A. C. Berry, The Fourier transform theorem, Journal of Mathematics and Physics, Massachusetts Institute of Technology, vol. 8 (1929), pp. 106-118, and N. Wiener, Laplacians and continuous linear functionals, Acta Szeged, vol. 3 (1927), pp. 7-16. 
value problem (29) may be treated on any closed $k$-dimensional space with a regular Riemann metric, if $\Delta u(x)$ is understood to be the corresponding Laplace-Beltrami operator. Hilbert has shown (explicitly for $k=2$ )* that in all such cases the solutions of (29) form a complete set of functions on the space. In case the given space has constant curvature the solutions of (29) are essentially the ultra-spherical functions. Expansions in ultra-spherical functions have been studied by many authors; $\dagger$ in the theory of Riesz summation, the exponent (12) again plays a decisive role. But the situation is slightly different. For expansions in ultra-spherical functions the localization property holds strictly only if $\delta \geqq 2 \alpha$, whereas if $2 \alpha>\delta>\alpha$ the antipode of the given point $x$ has also some influence.

The similarity of results relating to Fourier integrals and Fourier series suggests the investigation of larger classes of expansions. It would not be very profitable to include generalized Fourier integrals, but it will be rather illuminating to consider Fourier series not only of periodic functions but of the larger class of almost periodic functions. On this larger basis it will be easier to understand why the Riesz summation of order (12) no longer obeys the localization property in case $k \geqq 2$, and why all the trouble with the "crossneighborhood" of the given point $x$ which figures so prominently in the theory of summation by rectangular means is entirely eliminated in the case of spherical summation.

The almost periodic functions we shall deal with will be the functions belonging to a Stepanoff class $S_{p}, p \geqq 1$. $\ddagger$ For our purposes we may characterterize these functions as follows. The function $f(x)$ belongs to $S_{p}$ if it belongs to the Lebesgue class $L_{p}$ on every finite interval, and if there exists a sequence of exponential polynomials $f^{n}(x)$, that is, of functions each of which is a finite sum of the form

$$
\sum a_{n_{1} \cdots n_{k}}^{(n)} e^{i\left(n_{1} x_{1}+\cdots+n_{k} x_{k}\right)}
$$

(the numbers $n_{1}, \cdots, n_{k}$ being arbitrary real numbers), such that, for every $k$-dimensional unit-sphere $E$ of radius 1 , and uniformly in all such spheres,

$$
\lim _{n \rightarrow \infty} \int \cdots \int_{E}\left|f(x)-f^{n}(x)\right|^{p} d x_{1} \cdots d x_{k}=0 .
$$

* D.Hilbert, Grundzüge einer allgemeinen Theorie der linearen Integralgleichungen, 1912, Chapter XVIII. Grouping of terms by spherical shells suggests itself naturally in multiple Fourier series and other expansions arising in mathematical physics, for instance, in the discussion of vibrations of rectangular membranes. See Rayleigh, Theory of Sound, vol. I, 1894, Chapter IX.

† See E. Kogbetliantz, Sommation des séries et intégrales divergentes par les moyennes arithmétiques et typiques, Mémorial des Sciences Mathématiques, Fasc. 51, 1931, pp. 70-73.

$\ddagger$ See A. S. Besicovitch, Almost Periodic Functions, Cambridge, 1932. 
An almost periodic function determines, and is determined by a Fourier series of the form (1), the numbers $n_{1}, \cdots, n_{k}$ being arbitrary real numbers.

We shall see that the formulas and results concerning spherical summation extend very naturally to almost periodic functions in general. This explains the absence of conditions involving "cross-neighborhoods." Such conditions imply discriminations between the various directions in the underlying space. But no such discriminations are likely to play a role with almost periodic functions, since the class of these functions is invariant with respect to affine transformations of the $x$-space.

\section{PART II. FourIER INTEGRALS}

In the present section $f(x)$ is a measurable function for which

$$
\int \cdots \int_{-\infty}^{\infty}|f(x)| d x_{1} \cdots d x_{k}<\infty
$$

and $S_{R}{ }^{\phi}(x)$ is defined by (25)-(27).

The function $f_{x}(t)$, which was defined by (20), is measurable, and

$$
\int_{0}^{\infty}\left|f_{x}(t)\right| t^{k-1} d t<\infty \text {. }
$$

Lemma 1. The formula

$$
S_{R}^{\phi}(x)=R \int_{0}^{\infty} f_{x}(t) H_{\phi}(t R) d t
$$

holds if

$$
\int_{0}^{\infty}|\phi(t)| t^{k-1} d t<\infty .
$$

In the proof we may assume $x=(0, \cdots, 0)$. Substituting (26) in the relation (27) and changing the order of integrations (which is justified by (33)) we obtain

$$
S_{R}^{\phi}(0)=\int \cdots \int_{-\infty}^{\infty} f(x) F(x) d x_{1} \cdots d x_{k}
$$

where

$$
F(x)=\frac{1}{(2 \pi)^{k}} \int \cdots \int_{-\infty}^{\infty} \phi\left(\frac{\nu}{R}\right) e^{-i\left(n_{1} x_{1}+\cdots+n_{k} x_{k}\right)} d x_{1} \cdots d x_{k} .
$$

By an established formula*

* S. Bochner, Vorlesungen über Fouriersche Integrale, 1932, p. 186, Satz 56. 


$$
\begin{aligned}
(2 \pi)^{k / 2} F\left(x_{1}, \cdots, x_{k}\right) & =t^{-l} \int_{0}^{\infty} \phi\left(\frac{u}{R}\right) u^{l+1} J_{l}(u t) d u \\
& =R t^{-k+1} H_{\phi}(t R),
\end{aligned}
$$

where $t^{2}=x_{1}^{2}+\cdots+x_{k}^{2}$. Substituting this in (34) and making the change of coordinates $x_{1}=t \xi_{1}, \cdots, x_{k}=t \xi_{k}$ we finally obtain

$$
S_{R}^{\phi}(0)=R \int_{0}^{\infty} f_{0}(t) H_{\phi}(t R) d t .
$$

LEMma 2. Let $\phi(t)$ be either the Riesz kernel $K_{\delta}(t)$ with $\delta>\alpha$, or a (complex) function in $0 \leqq t<\infty$ having the following properties:

(i) The inequality

$$
\int_{0}^{\infty}|\phi(t)| t^{k-1} d t<\infty \quad(k-1=2 l+1)
$$

holds.

(ii) If $g$ is the integer defined by

$$
\frac{1}{2}>l-g \geqq-\frac{1}{2},
$$

then $\phi(t)$ has $(g+2)$ derivatives in $0 \leqq t<\infty$, each bounded in some neighborhood of $t=0$, such that ${ }^{*}$

$$
\varlimsup_{u \rightarrow \infty}\left|\phi^{(\gamma)}(u) u^{\lambda}\right|<\infty, \quad \quad \int_{0}^{\infty}\left|\phi^{(\gamma)}(u) u^{\lambda}\right| d u<\infty
$$

for

$$
\gamma=0,1, \cdots, g+2, \quad 0 \leqq \lambda \leqq l+\frac{1}{2} .
$$

Under these circumstances, $H_{\phi}(c)$ has the following properties:

$$
\begin{aligned}
\left|H_{\phi}(c)\right| & \leqq A_{1} c^{k-1} . \\
\left|H_{\phi}(c)\right| & \leqq \frac{A_{2}}{c^{1+\kappa}} \\
R \int_{0}^{\infty} H_{\phi}(t R) d t & =\phi(0) 2^{l} \Gamma(l+1),
\end{aligned}
$$

where $A_{1}, A_{2}, \kappa$ are positive constants independent of $c$.

For $\phi(t)=K_{\delta}(t)$ we have (18); (39) and (40) follow immediately, since $J_{\nu}(c)=O\left(c^{\nu}\right)$ as $c \rightarrow 0$, and $=O\left(c^{-1 / 2}\right)$ as $c \rightarrow \infty$. As to (41), see Watson, loc. cit., p. 391, formula (1).

* The conditions (38) are redundant for some values of $\gamma$ and $\lambda$. 
Now let $\phi(t)$ satisfy hypothesis (i). Since $\left|J_{l}(u c)\right| \leqq A(u c)^{l}$, we have

$$
\left|\int_{0}^{\infty} \phi(u) u^{l+1} J_{l}(u c) d u\right| \leqq A c^{l} \int_{0}^{\infty}|\phi(u)| u^{2 l+1} d u,
$$

and this proves (39). As to (40), what we have to prove is

$$
\int_{0}^{\infty} \phi\left(\frac{u}{c}\right) u^{l+1} J_{l}(u) d u=O\left(c^{-\kappa}\right), \quad c \rightarrow \infty .
$$

We shall prove more, namely,

LEMMA 3. If $l$ is any real number $\geqq-\frac{1}{2}$, and if a function $\phi(t)$ satisfies hypothesis (ii) of Lemma 2, then (42) holds.

We shall first treat the case $\frac{1}{2}>l \geqq-\frac{1}{2}$, that is, $g=0$. We observe that $u^{l+1} J_{l}(u)$ is the derivative of $u^{i+1} J_{l+1}(u)$ and that the function

$$
P(u)=\int_{0}^{u} u^{l+1} J_{l+1}(u) d u
$$

has the properties

$$
\begin{array}{ll}
P(u)=O\left(u^{2 l+3}\right)=O\left(u^{l+1 / 2}\right) & u \rightarrow 0, \\
P(u)=O\left(u^{l+1 / 2}\right) & u \rightarrow \infty .
\end{array}
$$

Making use of the various relations constituting (38) we can readily justify the following integrations by parts:

$$
\begin{aligned}
\int_{0}^{\infty} \phi\left(\frac{u}{c}\right) u^{l+1} J_{l}(u) d u & =-\frac{1}{c} \int_{0}^{\infty} \phi^{\prime}\left(\frac{u}{c}\right) u^{l+1} J_{l+1}(u) d u \\
& =\frac{1}{c^{2}} \int_{0}^{\infty} \phi^{\prime \prime}\left(\frac{u}{c}\right) P(u) d u .
\end{aligned}
$$

Majorizing $P(u)$ by $u^{l+1 / 2}$ in the whole interval $(0, \infty)$, we obtain (42) with $\kappa=\frac{1}{2}-l$. This disposes of the case $g=0$. The case of an arbitrary integer $g>0$ can now be dealt with by a process of induction, the induction working from the pair of values $(g, l)$ to the pair of values $(g+1, l+1)$. In fact,

$$
\begin{aligned}
\int_{0}^{\infty} \phi\left(\frac{u}{c}\right) u^{l+2} J_{l+1}(u) d u=\int_{0}^{\infty} \phi\left(\frac{u}{c}\right) u^{2 l+2} \frac{J_{l+1}(u)}{u^{l}} d u \\
=\int_{0}^{\infty} \frac{d}{d u}\left(\phi\left(\frac{u}{c}\right) u^{\imath \iota+2}\right) \frac{J_{l}(u)}{u^{l}} d u=\int_{0}^{\infty} \psi\left(\frac{u}{c}\right) u^{l+1} J_{l}(u) d u,
\end{aligned}
$$

where

$$
\psi(u)=(2 l+2) \phi(u)+u \phi^{\prime}(u)
$$


and the condition (38) is so arranged that if $\phi(u)$ satisfies it for $\left(g_{0}+1, l_{0}+1\right)$, $\psi(u)$ satisfies it for $\left(g_{0}, l_{0}\right)$. This completes the proof of Lemma 3, and therefore, the proof of $(40)$.

Relation (41) will follow from the Fourier-Hankel inversion formula* which if applied to the function $\phi(u) u^{l+1 / 2}$ reads

$$
\int_{0}^{\infty} \frac{J_{l}(c x)}{(c x)^{l}} d c \int_{0}^{\infty} \phi(u)(u c)^{l+1} J_{l}(u c) d u=\phi(x)
$$

or

$$
\int_{0}^{\infty} \frac{J_{l}(c x)}{(c x)^{l}} H_{\phi}(c) d c=\phi(x) .
$$

Since $J_{l}(c x) \cdot(c x)^{-l}$ is boundedly convergent if $x \rightarrow 0$, and $H_{\phi}(c)$ is absolutely integrable in $(0, \infty)$ on account of (39) and (40), we may deduce from (44) the relation

$$
\int_{0}^{\infty} H_{\phi}(c) d c=\phi(0) \cdot 2^{l} \Gamma(l+1),
$$

which is the same as (41).

TheOREM I. Let $\phi(t)$ be a function as in Lemma 2 for which $\phi(0)=1$. If for a given function $f(x)$ and $a$ fixed point $x$ there exists the limit

or, more generally, if

$$
\lim _{t \rightarrow 0} f_{x}(t)=s,
$$

$$
\lim _{t \rightarrow 0} t^{-k} \int_{0}^{t} t^{k-1}\left|f_{x}(t)-s\right| d t=0,
$$

then

$$
\lim _{R \rightarrow \infty} S_{R}^{\phi}(x)=2^{l} \Gamma(l+1) \cdot s .
$$

From (10) and (41) follows

$$
S_{R}^{\phi}(x)-2^{l} \Gamma(l+1) \cdot s=R \int_{0}^{\infty} g(t) H_{\phi}(t R) d t,
$$

where

$$
g(t)=f_{x}(t)-s .
$$

* See Watson, loc. cit., p. 456, $\$ 144$. 
In (48) we split the interval of integration in $\left(0, R^{-1}\right)$ and $\left(R^{-1}, \infty\right)$. Using (39) for $t R \leqq 1$ and (40) for $t R>1$, we see that (48) may be majorized by the sum of

$$
R^{k} F\left(R^{-1}\right)
$$

and

$$
R^{-\kappa} \int_{R^{-1}}^{\infty} t^{-\kappa-k} d F(t)
$$

where

$$
F(t)=\int_{0}^{t}|g(t)| t^{k-1} d t
$$

By (46) and (32),

$$
\begin{aligned}
F(t) & =o\left(t^{k}\right), \quad \text { as } t \rightarrow 0, \\
& =O\left(t^{k}\right), \quad \text { as } t \rightarrow \infty ;
\end{aligned}
$$

from this it follows easily that (50) and (51) both converge to 0 as $R \rightarrow \infty$.

Theorem I covers the Riesz kernel $\phi(t)=K_{\delta}(t)$ only for $\delta>\alpha$. Now let $\delta=\alpha-\beta, 0 \leqq \beta<1$. Since, up to a multiplicative constant, $H_{\phi}(t)$ has the value

$$
c^{l-\delta} J_{\delta+l+1}(c),
$$

we again have the relations (39) and (41), the integral in the latter relation being conditionally convergent at $\infty$; whereas instead of (40) we shall use, for $t R \geqq 1$, the relation

$$
H_{\delta}(t R)=A_{k \delta} R^{\beta-1} \frac{\cos (t R-(k+\beta) \pi / 2)}{t^{1-\beta}}+O\left((t R)^{\beta-2}\right)
$$

in which $A_{k \delta}$ is independent of $t$ and $R$. By Lemma 1 in connection with (41) we again have relation (48) and we again put

$$
R \int_{0}^{\infty} g(t) H_{\phi}(t R) d t=R \int_{0}^{R^{-1}}+R \int_{R^{-1}}^{\infty}=J_{1}(R)+J_{2}(R) .
$$

On account of (39) the mere condition (46) is again sufficient to secure the relation $\lim _{R \rightarrow \infty} J_{1}(R)=0$. But the previous estimation of $J_{2}(R)$ no longer holds true. If we substitute the value (54) for $H_{\delta}(t R)$ in $J_{2}(R)$ then the previous estimation applies only to the contribution arising from the error term. Thus we obtain the following 
THEOREM II. Let $\delta=\alpha-\beta, 0 \leqq \beta<1$. If $f_{x}(t)$ satisfies candition (46), then the limit relation

$$
\lim _{R \rightarrow \infty} S_{R}^{\delta}(x)=2^{l} \Gamma(l+1) \cdot s
$$

holds if, and only if,

$$
\lim _{R \rightarrow \infty} R^{\beta} \int_{R^{-1}}^{\infty}\left(f_{x}(t)-s\right) \frac{\cos (t R-(k+\beta) \pi / 2)}{t^{1-\beta}} d t=0 .
$$

For any $\epsilon>0$ and $0 \leqq \beta<1$,

$$
R^{\beta} \int_{\epsilon}^{\infty} \frac{\cos (t R-(k+\beta) \pi / 2)}{t^{1-\beta}} d t=\int_{\epsilon R}^{\infty} \frac{\cos (u-(k+\beta) \pi / 2)}{u^{1-\beta}} d u \rightarrow 0
$$

as $R \rightarrow \infty$.

On the other hand, for $\beta>0$, the assumption (32) does not imply

$$
R^{\beta} \int_{\epsilon}^{\infty} f_{x}(t) \frac{\cos (t R-(k+\beta) \pi / 2)}{t^{1-\beta}} d t \rightarrow 0 \quad \text { as } R \rightarrow \infty .
$$

In fact, for $\beta>0$, (58) need not hold even for a function $f(x)$ that vanishes outside a finite interval. However, for $\beta=0$, it is immediately seen that (32) implies (58) for $k>1$. Combining this with the familiar result in case $k=1$, we obtain the following

THEOREM III. If $f_{x}(t)$ satisfies condition (46) then the limit relation

$$
\lim _{R \rightarrow \infty} S_{R}^{\alpha}(x)=2^{\imath} \Gamma(l+1) \cdot s
$$

holds if, and only if, for some (or every) $\epsilon>0$,

$$
\lim _{R \rightarrow \infty} \int_{R^{-1}}^{\epsilon}\left(f_{x}(t)-s\right) \frac{\cos (t R-k \pi / 2)}{t} d t=0 .
$$

Thus for the validity of (59) it is sufficient that $g(t)=f_{x}(t)-s$ satisfies the conditions of Lebesgue

$$
\int_{0}^{\eta}|g(t)| t^{k-1} d t=o\left(\eta^{k}\right), \quad \int_{\eta}^{\epsilon} \frac{|g(t)-g(t+\eta)|}{t} d t \rightarrow 0 \quad \text { as } \eta \rightarrow 0 .
$$

For $\delta \geqq \alpha$, the relation (55) depends only on the behaviour of $f(x)$ in a neighborhood of the given point $x$; this is no longer the case for $\delta<\alpha$.

For $\delta<\alpha$, criteria for the validity of (55) will of necessity involve the behaviour of $f_{x}(t)$ in its entire interval $0 \leqq t<\infty$. For $0<\beta<1$, if (46) holds, it 
is sufficient to find conditions for the validity of (56). By classical methods it is easy to find that, using (46), (56) will hold if

$$
R^{\beta} \int_{R^{-1}}^{\infty} \frac{|g(t)-g(t+\pi / R)|}{t^{1-\beta}} d t \rightarrow 0 \quad \text { as } R \rightarrow \infty .
$$

This in its turn, will be satisfied if, for instance, $g(t)$ is the Lebesgue integral of a function $g^{\prime}(t)$ in every finite interval $0 \leqq t \leqq t_{0}$ such that

$$
\int_{1}^{\infty} \frac{\left|g^{\prime}(t)\right|}{t^{1-\beta}} d t<\infty
$$

$g(t)$ thus being absolutely continuous in $0 \leqq t \leqq t_{0}$, this condition implies the existence of the limit $g(+0)$, which is much more special than (46). Altogether we obtain the following criterion. For $0<\beta<1$, (55) will hold, and s will be $f_{x}(+0)$, if $f_{x}(t)$ is absolutely continuous in every finite interval $0 \leqq t \leqq t_{0}$, and

$$
\int_{1}^{\infty} \frac{\left|f_{x}^{\prime}(t)\right|}{t^{1-\beta}} d t .<\infty
$$

For $\delta<\alpha-1$, it is easy to find criteria for the validity of (55) involving the existence of derivatives of higher than the first order for the function $f_{x}(t)$. But there would not be much to be gained from a detailed analysis. The interesting case $\delta=0$ has been treated elsewhere.*

\section{PART III. FourIer SERIES}

LeMma 4. Corresponding to any dimension number $k$, any $p \geqq 1$, and any $\epsilon>0$, there exists a number $A=A(k, p, \epsilon)$ with the following property: if $f(x)=f\left(x_{1}, \cdots, x_{k}\right)$ belongs to class $L_{p}$ over every finite interval, and if there exists a number $B$ such that

$$
\int_{E}|f(x)|^{p} d x_{1} \cdots d x_{k} \leqq B
$$

for every $k$-dimensional sphere $E$ of radius 1 , then, for every point $x$,

$$
\begin{aligned}
& \int_{t}^{t+1}\left|f_{x}(t)\right|^{p} d t \leqq A B, \quad t \geqq \epsilon, \\
& \int_{0}^{1}\left|f_{x}(t)\right|^{p} t^{k-1} d t \leqq A B .
\end{aligned}
$$

Moreover

$$
\lim _{\epsilon \rightarrow 0} \int_{0}^{\epsilon}\left|f_{x}(t)\right|^{p} t^{k-1} d t=0 .
$$

* See S. Bochner, loc. cit. (footnote on p. 179). 
The proof follows easily from the relation

$$
\int_{a}^{b}\left|f_{x}(t)\right|^{p} t^{k-1} d t \leqq \omega_{k} \int_{S}|f(x)|^{p} d x_{1} \cdots d x_{k},
$$

where $\omega_{k}$ is a number depending only on $k$, and $S$ is the spherical shell with the radii $a$ and $b$, and its center at the point $x$.

LEMMA 5. Let $f(x) ; f^{1}(x), f^{2}(x), \cdots$ be a sequence of functions each belonging to class $L_{p}$ over every finite domain, and let

$$
\lim _{n \rightarrow \infty} \int_{E}\left|f(x)-f^{n}(x)\right|^{p} d x_{1} \cdots d x_{k}=0
$$

uniformly in all unit spheres $E$.

Then, uniformly in $\epsilon \leqq t<\infty$ and all $x$,

$$
\lim _{n \rightarrow \infty} \int_{t}^{t+1}\left|f_{x}(t)-f_{x}^{i}(t)\right|^{p} d t=0,
$$

and, for every $\epsilon>0$, uniformly in $x \equiv\left(x_{1}, \cdots, x_{k}\right)$,

$$
\lim _{n \rightarrow \infty} \int_{0}^{\epsilon}\left|f_{x}(t)-f_{x}^{n}(t)\right|^{p} t^{k-1} d t=0 .
$$

Lemma 5 is an immediate consequence of Lemma 4.

THEOREM IV. Let $\phi(t)$ be absolutely continuous over every finite range, and let

$$
\int_{0}^{\infty}|\phi(t)| t^{l+1 / 2} d t<\infty .
$$

Then the formula

$$
S_{R}^{\phi}(x)=R \int_{0}^{\infty} f_{x}(t) H_{\phi}(t R) d t
$$

holds for every (finite) exponential polynomial

$$
\sum a_{n_{1} \cdots n_{k}} e^{i\left(n_{1} x_{1}+\cdots+n_{k} x_{k}\right)}
$$

the numbers $n_{1}, \cdots, n_{k}$ being arbitrary real numbers.

It is sufficient to consider an exponential monomial (21). Formula (10) follows from a combination of (22), (23) with (43)-(45).

THeOREM V. If $\phi(t)$ is a function as in Lemma 2, then (10) holds for every almost periodic function of a Slepanoff class.

Let 


$$
f(x) \sim \sum a_{n_{1} \cdots n_{k}} e^{i\left(n_{1} x_{1}+\cdots+n_{k} x_{k}\right)}
$$

be a function of class $S_{1}$, and let

$$
f^{n}(x)=\sum a_{n_{1} \cdots n_{k}}^{(n)} e^{i\left(n_{1} x_{1}+\cdots+n_{k} x_{k}\right)} \quad(n=1,2, \cdots)
$$

be a sequence of exponential polynomials approximating $f(x)$ in the sense of Lemma 5, with $p=1$. This approximation implies

$$
\lim _{n \rightarrow \infty} a_{n_{1} \cdots n_{k}}^{(n)}=a_{n_{1} \cdots n_{k}} .
$$

If

$$
S_{R}^{\phi}\left(x ; f^{n}\right)=\sum \phi\left(\frac{\nu}{R}\right) a_{n_{1} \cdots n_{k}}^{(n)} e^{i\left(n_{1} x_{1}+\cdots+n_{k} x_{k}\right)},
$$

then, by Theorem IV,

$$
S_{R}^{\phi}\left(x ; f^{n}\right)=R \int_{0}^{\infty} f_{x}^{n}(t) H_{\phi}(t R) d t .
$$

Let now $n \rightarrow \infty$ in (72), $R$ being fixed. We split the integral on the right into $\int_{0}^{1}$ and $\int_{1}^{\infty}$. In the first part we may majorize $H_{\phi}(t R)$ by $t^{k-1}$, in the second part by $t^{-1-x}$ (see (39), (40)). Applying (67) to the first part and (66) to the second part, we find that the right side of (72) converges towards

$$
R \int_{0}^{\infty} f_{x}(t) H_{\phi}(t R) d t
$$

uniformly in $x$. Each function $S_{R}{ }^{\phi}\left(x ; f^{n}\right)$ is almost periodic. We have just seen that these functions converge uniformly in $x$. The limit function is again almost periodic and its Fourier series is the formal limit of the right side of (71). On account of (70) the resulting function has the Fourier series

$$
\sum \phi\left(\frac{\nu}{R}\right) a_{n_{1} \cdots n_{k}} e^{i\left(n_{1} x_{1}+\cdots+n_{k} x_{k}\right)} .
$$

It is therefore well justified to denote the function itself by $S_{R}^{\phi}(x)$, and the proof of our theorem is completed. We observe that $S_{R}{ }^{\phi}(x)$ is not only a Stepanoff function but a uniformly continuous almost periodic function in the original sense of $\mathrm{H}$. Bohr.

ThEOREM VI. Theorem I holds also for almost periodic functions of the Stepanoff class.

The proof of Theorem I goes through with the slight change that (52) follows now from (63) instead of from (32). 
But if we apply the Riesz kernel $\phi(t)=K_{\alpha}(t)$ with the critical exponent (12), the series behave differently from the integrals. For $k=1$, in which case $\alpha=0$, it is true that the relation (59) depends only on the behaviour of $f(x)$ in a neighborhood of $x$, at least for all pure periodic functions; this is the content of the well known localization theorem of Riemann. But for every $k \geqq 2$ this is no longer true, even for pure periodic functions, as will appear from the subsequent Theorem VII. Throughout the remainder of this section we shall assume $k \geqq 2$.

We shall denote a general lattice point $\left(m_{1}, \cdots, m_{k}\right)$ by $M$. We denote these points in some fixed order by $M_{1}, M_{2}, M_{3}, \cdots$. Furthermore we introduce, corresponding to every point $M_{p}$, the function

$$
\lambda_{p}(h)=\left[\left(m_{1}-h_{1}\right)^{2}+\cdots+\left(m_{k}-h_{k}\right)^{2}\right]^{1 / 2}
$$

of the real variables $h_{1}, \cdots, h_{k}$.

Lemma 6. For any finite sequence of constants $c_{1}, \cdots, c_{q}$ which do not vanish simultaneously, the points $h=\left(h_{1}, \cdots, h_{k}\right)$ for which

$$
\sum_{p=1}^{q} c_{p} \lambda_{p}(h)=0
$$

are nowhere dense in the Euclidean h-space.

The function $\lambda_{p}(h)$ is analytic at every point $h$, with the only exception of the point $h=M_{p}$ for which this is not the case. One of the numbers $c_{p}$ is $\neq 0$. Therefore the function on the left side of (76) is certainly singular in at least one point and, consequently, not identically zero. But the zeros of such an analytic function form a (closed) nowhere dense set.

Lemma 7. There exists an everywhere dense set of points $h$ for which the sequence of numbers

$$
\lambda_{1}(h), \lambda_{2}(h), \cdots
$$

is linearly independent with reference to integer (or rational) coefficients.

The desired set is complementary to the set of zeros of all functions $\sum_{p=1}^{q} c_{p} \lambda_{p}(h)$ whose coefficients $c_{1}, \cdots, c_{q}$ are integers. But the complementary set to an enumerable number of nowhere dense sets is everywhere dense.

Lemma 8. If $h$ is not a latlice point and $\beta>0$, then the sum

$$
C_{\beta}(\rho)=\sum_{p=1}^{\infty} \frac{e^{2 \pi i \rho} \backslash_{p}^{(h)}}{\lambda_{p}(h)^{k+\beta}}
$$

converges in $-\infty<\rho<\infty$. For every $\lambda$ the expression 


$$
\frac{1}{R} \int_{1}^{R} C_{\beta}(\rho) e^{-2 \pi i \rho \lambda} d \rho
$$

tends to a limit as $R \rightarrow \infty$, uniformly in $0<\beta \leqq \beta_{0}$ (where $\beta_{0}$ is any positive number). This limit has the value

$$
\left\{\begin{array}{cl}
0 & \text { if } \lambda \neq \lambda_{1}(h), \lambda_{2}(h), \cdots, \\
\lambda_{p}(h)^{-k-\beta} & \text { if } \lambda=\lambda_{p}(h) .
\end{array}\right.
$$

It is immediately scen that $\sum_{p=1}^{\infty} \lambda_{p}(h)^{-k-\beta}$ is convergent for $\beta>0$, that

$$
\sum_{\lambda_{p}(h) \neq \lambda} \frac{\lambda_{p}(h)^{-k-\beta}}{\lambda_{p}(h)-\lambda}
$$

converges uniformly in $0<\beta \leqq \beta_{0}$, and that

$$
\begin{aligned}
\left|\frac{1}{R} \int_{1}^{R} e^{2 \pi i \rho\left(\lambda_{p}(h)-\lambda\right)} d \rho\right| \leqq \frac{2}{R\left|\lambda_{p}(h)-\lambda\right|} & \text { if } \lambda \neq \lambda_{1}(h), \lambda_{2}(h), \cdots, \\
\frac{1}{R} \int_{1}^{R} e^{2 \pi i \rho\left(\lambda_{p}(h)-\lambda\right)} d \rho=1-\frac{1}{R} & \text { if } \lambda=\lambda_{p}(h) .
\end{aligned}
$$

The assertions of our lemma easily follow if we substitute (78) in (79) and interchange the order of summation and integration.

Lrмма 9. If $h$ is not a lattice point the function

$$
\Phi_{k}(h)=\sum_{\nu \leqq h}\left(1-\begin{array}{c}
\nu^{2} \\
R^{2}
\end{array}\right)^{\alpha} e^{-2 \pi i\left(n_{1} h_{1}+\cdots+n_{k} h_{k}\right)}, \quad \nu^{2}=n_{1}{ }^{2}+\cdots+n_{k}{ }^{2},
$$

has the following property. For each $\lambda$ the limit

$$
\lim _{R \rightarrow \infty} \frac{1}{R} \int_{0}^{R} \Phi_{\rho}(h) e^{-2 \pi i \rho \lambda} d \rho
$$

exists and cquals



For $\beta>0$ the function

$$
\Phi_{k}^{\beta}(h)=R^{\beta} \sum_{\nu \leqq l}\left(1-\frac{\nu^{2}}{R^{2}}\right)^{\alpha+\beta} e^{-2 \pi i\left(n_{1} h_{1}+\cdots+n k k_{k}\right)}
$$

has the value 


$$
\frac{\Gamma(\alpha+1+\beta)}{\pi^{\alpha+\beta}} R^{1 / 2} \sum_{p=1}^{\infty} \frac{J_{k+\beta-1 / 2}\left(2 \pi R\left(\lambda_{p}(h)\right)\right.}{\lambda_{p}(h)^{k+\beta-1 / 2}}
$$

provided $h$ is not a lattice point.* For $R \geqq 1$ this is, uniformly in $0<\beta \leqq \beta_{0}$,

$$
\frac{\Gamma(\alpha+1+\beta)}{2^{1 / 2} \pi^{k / 2+\beta}}\left[e^{-i k \pi / 2} C_{\beta}(R)+e^{i k \pi / 2} C_{\beta}(-R)\right]+O\left(R^{-1}\right) .
$$

Since (81) is the limit of (83) as $\beta \rightarrow 0$, Lemma 9 is a consequence of Lemma 8.

LEMMA 10. If the point $h$ is such that the sequence (77) is linearly independent with reference to integer coefficients, then

$$
\limsup _{R \rightarrow \infty}\left|\Phi_{R}(h)\right|=+\infty \text {. }
$$

The proof follows, on the basis of Lemma 9, from the following lemma by reductio ad absurdum.

LEMMA 11. $\dagger$ Let $f(x)$ be a real bounded measurable function in $0<x<\infty$ for which

$$
a(\Lambda)=\lim _{T \rightarrow \infty} \frac{1}{T} \int_{0}^{T} f(x) e^{-i \Delta x} d x
$$

exists for every $\Lambda$. Then a $(\Lambda)$ is different from zero for an at most enumerable set of numbers $\Lambda$ which we shall denote by $\Lambda_{1}, \Lambda_{2}, \ldots$. If the non-negative numbers

$$
\left|\Lambda_{1}\right|,\left|\Lambda_{2}\right|, \cdots
$$

are linearly independent with reference to integer coefficients, then

$$
\left|a\left(\Lambda_{1}\right)\right|+\left|a\left(\Lambda_{2}\right)\right|+\cdots<\infty .
$$

That $a(\Lambda)$ vanishes only for an enumerable number of values follows readily from Bessel's inequality which holds in this case. $f$ Denote the numbers (88) by $\mu_{1}, \mu_{2}, \cdots$. On putting

$$
K(x)=1+\frac{e^{i x}+e^{-i x}}{2}=1+\cos x \geqq 0,
$$

form for any real numbers $\alpha_{1}, \cdots, \alpha_{q}, q \geqq 2$, the conposite kernel

* See, for instance, J. R. Wilton, The lattice points of an n-dimensional ellipsoid, Journal of the London Mathematical Society, vol. 2 (1927), pp. 227-233; esp. p. 229.

$\dagger$ This lemma was stated without proof in a slightly different wording in S. Bochner, $\ddot{U} b e r$ Fourier-Reihen von fastperiodischen Funktionen, Sitzungsberichte der Berliner Mathematiker Gesellschaft, vol. 26 (1927), p. 54.

‡ See, for instance, M. Fekete, On generalized Fourier series with the non-negative coefficients, Proceedings of the London Mathematical Society, vol. 39 (1935), p. 321, footnote. 


$$
\begin{aligned}
K_{q}(x) & =\prod_{p=1}^{q} K\left(\mu_{p} x-\alpha_{p}\right) \\
& =1+\frac{1}{2} \sum_{p=1}^{q}\left(e^{i \mu_{p} x} e^{-i \alpha_{p}}+e^{-i \mu_{p} x} e^{i \alpha_{p}}\right)+R_{q}(x) .
\end{aligned}
$$

$R_{q}(x)$ is an exponential polynomial whose exponents are of the form $\epsilon_{1} \mu_{1}+\epsilon_{2} \mu_{2}+\cdots+\epsilon_{q} \mu_{q} ; \epsilon_{p}=0, \pm 1$, and at least two coefficients $\epsilon_{p}$ are different from zero. Owing to our assumption about the linear independence of $\mu_{1}, \mu_{2}, \cdots$, the exponents occurring in $R_{q}(x)$ are all different from $\pm \mu_{1}, \pm \mu_{2}, \cdots$. Hence*

$$
\lim _{T \rightarrow \infty} \frac{1}{T} \int_{0}^{T} f(t) K_{q}(t) d t=\frac{1}{2} \sum_{p=1}^{q}\left(a\left(\mu_{p}\right) e^{i \alpha_{p}}+a\left(-\mu_{p}\right) e^{-i \alpha_{p}}\right) .
$$

The absolute value of the left side is not greater than

$$
M=\underset{0<x<\infty}{1 . \mathrm{u} . \mathrm{b} .}\left|f^{\prime}(x)\right| \text {. }
$$

Hence

$$
\left|\sum_{p=1}^{q} a\left(\mu_{p}\right) e^{i \alpha_{p}}+a\left(-\mu_{p}\right) e^{-i \alpha_{p}}\right| \leqq M .
$$

Since $f(x)$ is real, $a\left(\mu_{p}\right)$ and $a\left(-\mu_{p}\right)$ are conjugate complex; for an appropriate choice of $\alpha_{p}$

$$
a\left(\mu_{p}\right) e^{i \alpha_{p}}+a\left(-\mu_{p}\right) e^{-i \alpha_{p}}=\left|a\left(\mu_{p}\right)\right|+\left|a\left(-\mu_{p}\right)\right| .
$$

Since $q$ may be any integer, (90) implies

$$
\sum_{p=1}^{\infty}\left\{\left|a\left(\mu_{p}\right)\right|+\left|a\left(-\mu_{p}\right)\right|\right\}<\infty,
$$

and this is the same relation as (89).

THEOREM VII. There exists a function $f(x)=f\left(x_{1}, \cdots, x_{k}\right)$ of class $L$ having the period 1 in each variable,

$$
f(x) \sim \sum a_{n_{1} \cdots n_{k}} e^{2 \pi i\left(n_{1} x_{1}+\cdots+n_{k} x_{k}\right)},
$$

and vanishing in a neighborhood of the origin $(0, \cdots, 0)$, such that

$$
\limsup _{R \rightarrow \infty}\left|\sum_{\nu \leqq R}\left(1-\frac{\nu^{2}}{R^{2}}\right)^{(k-1) / 2} a_{n_{1} \cdots n_{k}}\right|=\infty \text {. }
$$

* S. Bochner, Beiträge zur Theorie der fastperiodischen Funktionen, I, Mathematische Annalen, vol. 96 (1926), pp. 133-134; H. Bohr, Fastperiodische Funktionen, Ergebnisse der Mathematik, Berlin, Springer, 1932, p. 76. 
The function of $R$ occurring in (92) can be written as

$$
\int_{0}^{1} \cdots \int_{0}^{1} f(x) \sum_{\nu \leqq R}\left(1-\frac{\nu^{2}}{R^{2}}\right)^{(k-1) / 2} e^{-2 \pi i\left(n_{1} x_{1}+\cdots+n_{k} x_{k}\right)} d x_{1} \cdots d x_{k}
$$

or, see (81),

$$
\int_{E} f(x) \Phi_{R}(x) d x_{1} \cdots d x_{k},
$$

where $E$ is a measurable set within the fundamental domain of periodicity outside of which $f(x)$ vanishes.

If our theorem were not true, then for every set $E$ omitting a neighborhood of the origin, (93) would be bounded as $R \rightarrow \infty$ for every function $f(x)$ of class $L$ on $E$. We are now going to refute this possibility. Consider on $E$ the Banach space $\subseteq$ of functions $f(x)$ belonging to class $L$, with the usual metric. For each $R,(93)$ defines a functional in $\subseteq$; we shall denote it by $F_{R}(f)$. The norm of this functional is

$$
\text { ess. l. u. b. }\left|\Phi_{R}(x)\right| \text {. }
$$

Since $\Phi_{R}(x)$ is continuous in $x$, if we assume $E$ to be closed, we can write for this

$$
\max _{x \in E}\left|\Phi_{R}(x)\right| .
$$

By a known theorem, if a set of functionals $F_{R}(f)$, is such that for every $f_{\epsilon} \subseteq$

$$
\limsup _{R \rightarrow \infty}\left|F_{R}(f)\right|<\infty \text {, }
$$

then also

$$
\underset{R \rightarrow \infty}{\lim \sup }\left\|F_{R}\right\|<\infty .^{*}
$$

But

$$
\limsup _{R \rightarrow \infty} \max _{x \in E}\left|\Phi_{R}(x)\right|<\infty
$$

implies

$$
\limsup _{R \rightarrow \infty}\left|\Phi_{R}(x)\right|<\infty, \quad x \in E .
$$

But it follows from Lemmas 7 and 10 that there are sets $E$ for which (95) does not hold in all points $x$. This proves our theorem.

\footnotetext{
* See S. Banach, Théorie des opérations linéaires, 1932, p. 80, Theorem 5.
} 
We are now going to make a few remarks concerning quadrangular partial sums of the form (24).

LEMma 11. If $\phi(t)$ is continuous in $0 \leqq t<\infty$, non-negative, monotonely nonincreasing, and if $\int_{0}^{\infty} \phi(t) d t<\infty$, then

$$
\lim _{R \rightarrow \infty} \frac{1}{R} \sum_{n=0}^{\infty} \phi\left(\frac{a}{R} n\right)=\frac{1}{a} \int_{0}^{\infty} \phi(t) d t .
$$

The proof follows immediately from

$$
\int_{n}^{n+1} \phi\left(\frac{a}{R} t\right) d t \leqq \phi\left(\frac{a}{R} n\right) \leqq \int_{n-1}^{n} \phi\left(\frac{a}{R} t\right) d t, \quad n \geqq 1 .
$$

THEOREM VIII. Let $\phi(t)$ have the following properties. It has two continuous derivatives in $0 \leqq t<\infty . \phi(t)$ and $\phi^{\prime \prime}(t)$ are non-negative and monotonely nonincreasing as $t$ increases. Furthermore

$$
\gamma=\int_{0}^{\infty} \phi(t) d t<\infty, \quad \gamma_{2}=\int_{0}^{\infty} \phi^{\prime \prime}(t) d t<\infty .
$$

Then, for $k \geqq 3$, there exists a function $f(x)$ of period 1 in each variable and vanishing in a neighborhood of the origin, such that

$$
\underset{R \rightarrow \infty}{\limsup }\left|\sigma_{R}^{\phi}(0)\right|=\infty \text {. }
$$

Since

where

$$
\sigma_{R}^{\phi}(0)=\int_{0}^{1} \cdots \int_{0}^{1} f(x) \sum_{\kappa=1}^{k} \Phi_{R}\left(x_{\kappa}\right) d x_{\kappa}
$$

$$
\Phi_{R}(t)=\sum_{-\infty}^{\infty} \phi\left(\frac{|n|}{R}\right) e^{-2 \pi i n t},
$$

it is sufficient to show the existence of one point $\left(x_{1}^{0}, \cdots, x_{k}^{0}\right) \not \equiv(0, \cdots, 0)$ $(\bmod 1)$ for which

$$
\underset{R \rightarrow \infty}{\lim \sup } \prod_{\kappa=1}^{k}\left|\Phi_{R}\left(x_{\kappa}\right)\right|=\infty .
$$

We choose the point $x_{1}^{0}=\cdots=x_{k-1}^{0}=0, x_{k}^{0}=\frac{1}{2}$. Since

$$
\Phi_{R}(0)=\phi(0)+2 \sum_{n=1}^{\infty} \phi\left(\frac{n}{R}\right)
$$

therefore, by Lemma 11, $\Phi_{R}(0)$ has asymptotically, the value $\gamma R$. On the other hand 


$$
\begin{aligned}
\Phi_{R}\left(\frac{1}{2}\right) & =\phi(0)-2 \phi\left(\frac{1}{R}\right)+2 \phi\left(\frac{2}{R}\right)-2 \phi\left(\frac{3}{R}\right)+\cdots \\
& =\sum_{n=0}^{\infty} \int_{0}^{R^{-1}} \int_{0}^{R^{-1}} \phi^{\prime \prime}\left(\frac{2 n}{R}+t_{1}+t_{2}\right) d t_{1} d t_{2} .
\end{aligned}
$$

Hence

$$
\sum_{n=1}^{\infty} \phi^{\prime \prime}\left(\frac{2 n}{R}\right) \leqq R^{2} \Phi_{R}\left(\frac{1}{2}\right) \leqq \sum_{n=0}^{\infty} \phi^{\prime \prime}\left(\frac{2 n}{R}\right)
$$

and therefore, by Lemma $11, \Phi_{R}\left(\frac{1}{2}\right)$ has the asymptotic value $\gamma_{2} R^{-1} / 2$. Thus, $\Pi_{k=1} \Phi_{R}\left(x_{k}^{0}\right)$ has, apart from a constant, the asymptotic value $R^{k-2}$ and consequently, is not bounded in $R$ if $k \geqq 3$.

Under Theorem VIII fall such smooth functions as $e^{-t}$, and $(1-t)^{\delta}$ for arbitrarily large values of $\delta$. It is true that there are large classes of smooth functions $\phi(t)$ for which the quadrangular sums (24) do obey the localization property. To the latter ones belong $e^{-t^{2}}$, and $\left(1-t^{2}\right)^{\delta}$ for $\delta>k$ (but not for $\delta<k)$. But this makes things only worse, in a way. In fact, by a general theorem* summation functions as $(1-t)^{\delta},\left(1-t^{2}\right)^{\delta}$ are absolutely equivalent in the case of circular summation, whereas for quadrangular summation this equivalence theorem is apparently lost.

\section{PART IV. Convergence AND Gap theorems}

We shall first give criteria for convergence and absolute convergence of the series (5). They will impose reasonable conditions on the function $f_{x}(t)$ alone, and no other explicit conditions on the function $f\left(x_{1}, \cdots, x_{k}\right)$ itself.

THEOREM IX. Let $f(x)$ be a function of class $L$ having the period $2 \pi$ in each variable. If for a fixed point $x$ the function $f_{x}(t)$ has $k$ derivatives which are all bounded in $0 \leqq t<\infty$, and if, for $k$ even, the kth derivative of $f_{x}(t)$ is continuous at $t=0$, then the series (5) converges toward $f_{x}(0)=f(x)$.

Since our theorem certainly holds for a constant function we may assume $f_{x}(0)=0$. For each integer $n$ we put

$$
a_{n}=a_{n}(x)=\sum_{n=n_{1}^{2}+\cdots+n_{k}^{2}} a_{n_{1} \cdots n_{k}} e^{i\left(n_{1} x_{1}+\cdots+n_{k} x_{k}\right)} .
$$

If $\delta$ exceeds $(k-1) / 2$, then, by Theorem $\mathrm{V}$,

$$
\sum_{n \leqq R^{2}}\left(1-\frac{n}{R^{2}}\right)^{\delta} a_{n}=R \int_{0}^{\infty} f_{x}(t) H_{\delta}(t R) d t .
$$

${ }^{*}$ G. H. Hardy, The second theorem of consistency for summable series, Proceedings of the London Mathematical Society, vol. 15 (1915), pp. 72-88. 
$H_{\delta}(t)$ has, for some $\kappa>0$, the asymptotic expansion

$$
\frac{e^{i t}}{t^{1+\kappa}} \sum_{n=0}^{\infty} A_{n} t^{-n}+\frac{e^{-i t}}{t^{1+\kappa}} \sum_{n=0}^{\infty} B_{n} t^{-n}
$$

Each of the functions

$$
P_{1}(t)=-\int_{t}^{\infty} H_{\delta}(t) d t, \quad P_{2}(t)=-\int_{t}^{\infty} P_{1}(t) d t, \cdots
$$

has the same property. Hence we may integrate by parts in (97), and the result is:

$$
\sum_{n \leq R^{2}}\left(1-\frac{n}{R^{2}}\right)^{\delta} a_{n}=\frac{b_{1}}{R}+\cdots+\frac{b_{k-1}}{R^{k-1}}+\frac{1}{R^{k}} Q(R)
$$

where

$$
Q(R)=\int_{0}^{\infty} f_{x}^{(k)}\left(\frac{t}{R}\right) P_{k}(t) d t .
$$

$P_{k}(t)$ is absolutely integrable over $0 \leqq t<\infty$, and $f_{x}^{(k)}(t)$ is bounded in this interval. Therefore

$$
Q(R)=O(1) \quad \text { as } R \rightarrow \infty .
$$

If, in addition, $f_{x}^{(k)}(t)$ is continuous at $t=0$, then

$$
Q(R) \rightarrow f_{x}^{(k)}(0) \cdot \int_{0}^{\infty} P_{k}(t) d t \quad \text { as } R \rightarrow \infty .
$$

Replacing $R^{2}$ by $\omega$ in (98) we obtain

(101) $\sum_{n \leqq \omega}(\omega-n)^{\delta} a_{n}=b_{1} \omega^{\delta-1 / 2}+b_{2} \omega^{\delta-1}+\cdots+b_{k-1} \omega^{\delta-(k-1) / 2}+\omega^{\delta-k / 2} Q\left(\omega^{1 / 2}\right)$

whereas the assertion of our theorem reads

$$
\sum_{n \leqq \omega} a_{n}=o(1) .
$$

We shall show that (102) follows from (101) in connection with (100) or (99) respectively.

We first treat the case when $k$ is an even number. The integer $\mu=k / 2$ exceeds $(k-1) / 2$, thus (101) holds for $\delta=\mu$. Hence

$$
\sum_{n \leqq \omega}(\omega-n)^{\mu} a_{n}=b_{1} \omega^{\mu-1 / 2}+b_{2} \omega^{\mu-1}+\cdots+b_{k-1} \omega^{1 / 2}+Q\left(\omega^{1 / 2}\right) .
$$

In order to deduce (102), we apply to both sides of (103) the operator 
(104)

$$
\begin{aligned}
\underset{\mu, \eta}{\Delta A} A(\omega) & =A(\omega)-\left(\begin{array}{l}
\mu \\
1
\end{array}\right) A(\omega+\eta)+\left(\begin{array}{l}
\mu \\
2
\end{array}\right) A(\omega+2 \eta)-\cdots \\
& =(-1)^{\mu} \int_{0}^{\eta} \cdots \int_{0}^{\eta} A^{(\mu)}\left(\omega+t_{1}+\cdots+t_{\mu}\right) d t_{1} \cdots d t_{\mu}
\end{aligned}
$$

for the special values

$$
\omega=n+\frac{1}{\mu+2}, \quad \eta=\frac{1}{\mu+2} .
$$

Since

$$
\underset{\mu, \eta}{\Delta} \sum_{n \leqq \omega}(\omega-n)^{\mu} a_{n}=(-1)^{\mu} \eta^{\mu} \sum_{n \leqq \omega} a_{n}
$$

and

$$
\underset{\mu, \eta}{\Delta}\left(b_{1} \omega^{\mu-1 / 2}+b_{2} \omega^{\mu-1}+\cdots\right)=O\left(\omega^{-1 / 2}\right),
$$

we have only to verify that

$$
\underset{\mu, \eta}{\Delta} Q\left(\omega^{1 / 2}\right)=o(1) .
$$

But this follows from the relations

$$
\begin{aligned}
& \underset{\mu, \eta}{\Delta}=\underset{\mu-1, \eta}{\Delta}(\Delta), \\
& \underset{1, \eta}{\Delta} Q\left(\omega^{1 / 2}\right)=o(1), \\
& \underset{\mu-1, \eta}{\Delta} o(1)=o(1),
\end{aligned}
$$

relation (110) being a consequence of (100).

When $k$ is an odd number we put $k=2 \mu+1$. Relation (101) holds for $\delta>\mu$; also, by (99), $Q\left(\omega^{1 / 2}\right)=O(1)$. Hence, for fixed $\epsilon>0$,

$$
\sum_{n \leqq \omega}(\omega-n)^{\mu+\epsilon} a_{n}=b_{1} \omega^{\mu+\epsilon-1 / 2}+\cdots+O\left(\omega^{\epsilon-1 / 2}\right) .
$$

We again take, on both sides of (112), the operator (104) for the special values (105), and we obtain

$$
\underset{\mu, \eta}{\Delta} \sum_{n \leqq \omega}(\omega-n)^{\mu+\epsilon} a_{n}=O\left(\omega^{\epsilon-1 / 2}\right)+O\left(\omega^{\epsilon-1 / 2}\right) .
$$

If $\epsilon$ is less than $\frac{1}{2}$, then the right side of (113) is $o(1)$; and, introducing the constants

$$
\alpha_{\nu}=\int_{0}^{\eta} \cdots \int_{0}^{\eta}\left(\nu+\eta+t_{1}+\cdots+t_{\mu}\right)^{\epsilon} d t_{1} \cdots d t_{\mu},
$$


we deduce from (113) the set of relations

$$
\alpha_{0} a_{n}+\alpha_{1} a_{n-1}+\cdots+\alpha_{n} a_{0}=o(1), \quad n \rightarrow \infty .
$$

Introducing the partial sums

$$
s_{n}=a_{0}+a_{1}+\cdots+a_{n}
$$

and the new constants

$$
\beta_{0}=\alpha_{0}, \beta_{1}=\alpha_{1}-\alpha_{0}, \beta_{2}=\alpha_{2}-\alpha_{1}, \cdots,
$$

we obtain the new relations

$$
\beta_{0} s_{n}+\beta_{1} s_{n-1}+\cdots+\beta_{n} s_{0}=o(1),
$$

whereas the assertion of our theorem reads

$$
s_{n}=o(1) \text {. }
$$

We consider the analytic function

$$
\Phi(z)=\beta_{0}+\beta_{1} z+\beta_{2} z^{2}+\cdots
$$

and the power series expansion

$$
\frac{1}{\Phi(z)}=\gamma_{0}+\gamma_{1} z+\gamma_{2} z^{2}+\cdots,
$$

and we shall prove that

$$
\sum_{n=0}^{\infty}\left|\gamma_{n}\right|<\infty .
$$

Relation (116) is an immediate consequence of (117), since the equations

$$
\beta_{0} s_{n}+\beta_{1} s_{n-1}+\cdots+\beta_{n} s_{0}=r_{n}
$$

have the unique solution

$$
s_{n}=\gamma_{0} r_{n}+\gamma_{1} r_{n-1}+\cdots+\gamma_{n} r_{0} .
$$

The proof of (117) will be based on the following two properties of the numbers (115):

$$
\begin{aligned}
& \beta_{n}=B n^{\epsilon-1}+O\left(n^{\epsilon-2}\right), \quad B \neq 0, \\
& \beta_{0}>\beta_{1}>\beta_{2}>\cdots \geqq 0 .
\end{aligned}
$$

In order to secure (119) it is sufficient to fix $\epsilon$ so small that $\rho^{\epsilon}>(\rho+1)^{\epsilon}-\rho^{\epsilon}$ for $\rho \geqq \eta$. Both properties can be easily derived from the definition (114).

The function $\Phi\left(e^{i \theta}\right)$ is continuous and different from zero for $\theta \neq 0$. This follows immediately from 


$$
(1-z) \Phi(z)=\beta_{0}-\left(\beta_{0}-\beta_{1}\right) z-\left(\beta_{1}-\beta_{2}\right) z^{2}-\cdots
$$

in connection with property (119). Property (118) can be rewritten in the form

hence

$$
\beta_{n}=C\left(\begin{array}{c}
n+\epsilon-1 \\
n
\end{array}\right)+O\left(n^{\epsilon-2}\right), \quad C \neq 0 ;
$$

$$
\Phi(z)=C(1-z)^{-\bullet}+\phi(z),
$$

where the function $\phi(z)$ is such that the Fourier series of $\phi\left(e^{i \theta}\right)$ is absolutely convergent. Since the Fourier series of $\left(1-e^{i \theta}\right)^{\epsilon}$ is also absolutely convergent, and $\phi\left(e^{i \theta}\right) \cdot\left(1-e^{i \theta}\right) \cdot$ is 0 for $\theta=0$, we conclude that the function

$$
\Psi\left(e^{i \theta}\right)=\left(1-e^{i \theta}\right)^{\epsilon} \Phi\left(e^{i \theta}\right) \equiv C+\phi\left(e^{i \theta}\right)\left(1-e^{i \theta}\right)^{\epsilon}
$$

is nowhere 0 and has an absolutely convergent Fourier series. By an important theorem of N. Wiener* the Fourier series of $\left\{\Psi\left(e^{i \theta}\right)\right\}^{-1}$ is also absolutely convergent, and so is the Fourier series of $\left\{\Phi\left(e^{i \theta}\right)\right\}^{-1}=\left(1-e^{i \theta}\right)\left\{\psi\left(e^{i \theta}\right)\right\}^{-1}$. But the numbers $\gamma_{n}$ are the Fourier coefficients of the latter function and thus (117) is proved.

TheOREM X. Let $f(x)$ be a function of class $L$ having the period $2 \pi$ in each variable. If for a fixed point $x$ the function $f_{x}(t)$, has $k+4$ derivatives which are all bounded in $0 \leqq t<\infty$, then the simple series (5) converges absolutely toward $f_{x}(0)=f(x)$.

The theorem asserts that, under the stated assumptions,

$$
\sum_{n=0}^{\infty}\left|a_{n}(x)\right|<\infty \text {. }
$$

Replacing $k$ by $k+4$ in the right side of (98) we obtain the relation

$$
\sum_{n \leqq \omega}(\omega-n)^{\delta} a_{n}=b_{1} \omega^{\delta-1 / 2}+b_{2} \omega^{\delta-1}+\cdots+O\left(\omega^{\delta-(k+4) / 2}\right) .
$$

Let $\mu$ denote the smallest integer exceeding $(k-1) / 2$, thus $\mu \leqq(k+1) / 2$. Putting $\delta=\mu$ in (121) and applying the operator (104) for the values (105) we obtain

$$
\sum_{n \leqq \omega} a_{n}=c_{1} \omega^{-1 / 2}+c_{2} \omega^{-1}+O\left(\omega^{-3 / 2}\right)+O\left(\omega^{(k+1) / 2-(k+4) / 2}\right) .
$$

\section{Hence}

* N. Wiener, Tauberian theorems, Annals of Mathematics, (2), vol. 33 (1932), p. 14. Compare also R. E. A. C. Paley and N. Wiener, Fourier Transforms in the Complex Domain, American Ma thematical Society Colloquium Publications, vol. 19, New York, 1934, p. 59. 


$$
a_{n}=a_{n}(x)=O\left(n^{-3 / 2}\right) .
$$

ThEOREM XI. Let $f(x)$ be an almost periodic function of class $S$ whose Fourier exponents $\left(n_{1}, \cdots, n_{k}\right)$ have no finite accumulation point, and let its Fourier series be written in the form

$$
\begin{gathered}
\sum_{n=0}^{\infty} a_{\lambda_{n}}(x) \\
\lambda_{1}<\lambda_{2}<\lambda_{3}<\cdots \rightarrow \infty,
\end{gathered}
$$

where

$$
a_{\lambda}(x)=\sum_{\lambda=n_{1}+\cdots+n_{k^{2}}} a_{n_{1} \cdots n_{k}} e^{i\left(n_{1} x_{1}+\cdots+n_{k} x_{k}\right)} .
$$

Suppose further that for a subsequence $\left\{\lambda_{n_{j}}\right\}$ of $\left\{\lambda_{n}\right\}$ and a constant $\sigma \geqq 0$,

$$
\left(\lambda_{n_{j}+1}-\lambda_{n_{j}}\right)^{-1}=O\left(\lambda_{n_{j}}^{\sigma}\right) \text {. }
$$

If, for a fixed point $x, f_{x}(t)$ has more than $(k+1)(1+\sigma)$ derivatives each of which is bounded in $0 \leqq t<\infty$, then

$$
\lim _{j \rightarrow \infty} \sum_{n=0}^{n_{j}} a_{\lambda_{n}}(x)=f(x) .
$$

We again assume $f(x)=0$. If $f_{x}(t)$ has $q$ derivatives, then, in analogy to (121),

$$
\sum_{\lambda_{n} \leqq \omega}\left(\omega-\lambda_{n}\right)^{\delta} a_{\lambda_{n}}(x)=b_{1} \omega^{\delta-1 / 2}+\cdots+O\left(\omega^{\delta-q / 2}\right) .
$$

Let $a>0$ be so chosen that for all large values of $j$

$$
\lambda_{n_{j+1}}-\lambda_{n_{j}} \geqq \mu a \lambda_{n_{j}}^{-\sigma},
$$

where $\mu$ is again the least integer exceeding $(k-1) / 2$. We apply to (124), with $\delta=\mu$, the operator (104) for the values

$$
\omega=\lambda_{n_{j}}, \quad \eta=a \lambda_{n_{j}}^{-\sigma}=a \omega^{-\sigma},
$$

and we obtain

$$
\eta^{\mu} \sum_{\lambda_{n} \leqq \omega} a_{\lambda_{n}}(x)=O\left(\eta^{\mu} \omega^{-1 / 2}\right)+O\left(\omega^{\mu-q / 2}\right)
$$

hence

$$
\sum_{\lambda_{n} \leqq \omega} a_{\lambda_{n}}(x)=o(1)+O\left(\omega^{\mu+\mu \sigma-q / 2}\right) .
$$

But for $q>(k+1)(1+\sigma)$ the last term is $o(1)$, and the proof of our theorem is completed. 
Theorems IX-XI give criteria for Riesz summability of order $\delta=0$. The following theorems will be concerned with Riesz summability for the critical exponent $\delta=\alpha$.

TheOREM XII. Let $f(x)$ be an almost periodic function of class $S_{p}, p>1$, whose Fourier exponents have no finite accumulation point. If for a given point $x$, $f_{x}(t)$ satisfies the (local) Lebesgue condition (61) then, for every $q>0$, no matter how large,

$$
\lim _{R \rightarrow \infty} \int_{R}^{R+1}\left|S_{\rho}^{\alpha}(x)-2^{l} \Gamma(l+1) \cdot s\right|^{q} d \rho=0 .
$$

Since the theorem is obviously true for a constant function we can assume $s=0$. Since the class $S_{p}$ is the more general the smaller $p$ is and relation (125) is the more general the larger $q$ is, we may assume in the proof of the theorem that

$$
q=\frac{p}{p-1}
$$

Let $f^{n}(x)$ be a sequence of exponential polynomials as in Lemma 5. They may be so chosen that no new Fourier exponents $\left(n_{1}, \cdots, n_{k}\right)$ arise. Since the Fourier exponents of the given function $f(x)$ have no finite point of accumulation, it is trivial that $S_{R}\left(x, f^{n}\right)$ (see (71)) tends to $S_{R}(x, f)$ as $n \rightarrow \infty$ for every $\delta \geqq 0$, in particular for $\delta=\alpha$.

By Lemmas 4 and 5,

$$
\begin{array}{r}
\lim _{n \rightarrow \infty} \int_{0}^{\epsilon}\left|f_{x}(t)-f_{x}^{n}(t)\right|^{p} t^{k-1} d t=0, \\
\int_{\epsilon}^{\infty}\left|\frac{f_{x}(t)}{t}\right|^{p} d t<\infty, \\
\lim _{n \rightarrow \infty} \int_{\epsilon}^{\infty}\left|\frac{f_{x}(t)}{t}-\frac{f_{x}^{n}(t)}{t}\right|^{p} d t=0
\end{array}
$$

and the condition (61) reads for $s=0$,

$$
\frac{1}{\eta^{k}} \int_{0}^{\eta} t^{k-1}\left|f_{x}(t)\right| d t \rightarrow 0, \quad \int_{\eta}^{\epsilon} \frac{\left|f_{x}(t)-f_{x}(t+\eta)\right|}{t} d t \rightarrow 0 \text { as } \eta \rightarrow 0 .
$$

By Theorem IV

$$
S_{R}^{\alpha}\left(x ; f^{n}\right)=R \int_{0}^{\infty} f_{x}^{n}(t) H_{\alpha}(t R) d t=P_{R}^{\alpha}\left(x, f^{n}\right)+Q_{R}^{\alpha}\left(x, f^{n}\right),
$$


where

$$
\begin{aligned}
& P_{R}^{\alpha}(x, f)=R \int_{0}^{\epsilon} f_{x}(t) H_{\alpha}(t R) d t \\
& Q_{R}^{\alpha}(x, f)=R \int_{\epsilon}^{\infty} f_{x}(t) H_{\alpha}(t R) d t .
\end{aligned}
$$

Since, for fixed $R, H_{\alpha}(t R)$ is majorized by $t^{k-1}$ in $0 \leqq t \leqq \epsilon$, it follows from (127), if we use it for $p=1$,

$$
\lim _{n \rightarrow \infty} P_{R}^{\alpha}\left(x, f^{n}\right)=P_{R}^{\alpha}(x, f) .
$$

Furthermore, using the asymptotic expansion

$$
R H_{\alpha}(t R) \sim \frac{e^{i t R}}{t} \sum_{0}^{\infty} \Lambda_{n}(t R)^{-n}+\frac{e^{-i t R}}{t} \sum_{0}^{\infty} B_{n}(t R)^{-n}
$$

which is valid for $t R \rightarrow \infty$, the argument leading to Theorem III shows that, under condition (130), $P_{l}{ }^{\alpha}(x, f) \rightarrow 0$ as $R \rightarrow \infty$. In particular,

$$
\lim _{R \rightarrow \infty} \int_{R}^{R+1}\left|P_{R}^{\alpha}(x, f)\right|^{q} d R=0 .
$$

The term $Q_{R}{ }^{\alpha}(x, f)$ is less simple. Using (128) and (134) we see as in the theory of Hankel transforms, ${ }^{*}$ that $Q_{R}{ }^{\alpha}(x, f)$ exists if the integral (132) is defined as a limit-in-mean, and that

$$
\int_{1}^{R}\left|Q_{R}^{a}(x, f)\right|^{a} d R<\infty
$$

In particular

$$
\lim _{R \rightarrow \infty} \int_{R}^{R+1}\left|Q_{R}^{\alpha}(x, f)\right|^{q} d R=0 .
$$

Furthermore, on account of (129),

$$
\lim _{n \rightarrow \infty} \int_{1}^{\infty}\left|Q_{R}^{\alpha}\left(x, f^{n}\right)-Q_{R}^{\alpha}(x, f)\right|^{q} d R=0 .
$$

Hence, for almost all $R$,

$$
S_{R}^{\alpha}(x, f)=P_{R}^{\alpha}(x, f)+Q_{R}^{\alpha}(x, f) .
$$

* See E. C. Titchmarsh, A note on Ilankel transforms, Journal of the London Mathematical Society, vol. 1 (1926), pp. 195-196. 
Relation (125) follows now from (135)-(137).

LEMMA 12. Let $f(x)$ be an almost periodic function of class $S$ whose Fourier exponents have no finite point of accumulation. Then the relation

$$
S_{R}^{\alpha}(x, f)=R \int_{0}^{\infty} f_{x}(t) H_{\alpha}(t R) d t
$$

holds in the following integrated form. If $A(R)$ is any absolutely continuous function in any finite interval $(0<) R_{1} \leqq R \leqq R_{2}$, then

$$
\int_{R_{1}}^{R_{2}} A(R) S_{R}^{\alpha}(x) d R=\int_{0}^{\infty} f_{x}(t) d t \int_{R_{1}}^{R_{2}} A(R) R H_{\alpha}(t R) d R .
$$

We again introduce approximating exponential polynomials $f^{n}(x)$. The relation (138) holds for $f_{x}^{n}(t)$, and since, for each $n$,

$$
\int_{0}^{\infty}\left|f_{x}^{n}(t)\right|\left|H_{\alpha}(t R)\right| d t<\infty
$$

uniformly in $R_{1} \leqq R \leqq R_{2},(139)$ is true for $f^{n}(x)$. Thus we have only to show that

$$
\text { (140) } \lim \int_{0}^{\infty} f_{x}^{n}(t) d t \int_{R_{1}}^{R_{2}} A(R) R H_{\alpha}(t R) d R=\int_{0}^{\infty} f_{x}(t) d t \int_{R_{1}}^{R_{2}} A(R) R H_{\alpha}(t R) d R \text {. }
$$

Now, using (134), it is easily seen that

$$
\int_{R_{1}}^{R_{2}} A(R) R H_{\alpha}(t R) d R=O\left(t^{-2}\right) \text { as } t \rightarrow \infty ;
$$

on the other hand

$$
\int_{R_{1}}^{R_{2}} A(R) R H_{\alpha}(t R) d R=O\left(t^{k-1}\right) \text { as } t \rightarrow 0 .
$$

Relation (140) follows now from a combination of (141), (142) with Lemma 5.

Lemma 13. Let $f(x)$ be an almost periodic function of class $S$ whose Fourier exponents have no finite point of accumulation. If, for, a given point $x, f_{x}(t)$ satisfies the (local) Lebesgue condition (61), then, for any $\gamma \geqq 0$, and any function $P(\rho)$ which has a bounded derivative in $-1 \leqq \rho \leqq 1$,

$$
\lim _{R \rightarrow \infty} \int_{-1}^{1} P(\rho)\left(1+\frac{\rho}{R}\right)^{\gamma} S_{R+\rho}^{\alpha}(x) d \rho=2^{l} \Gamma(l+1) \cdot s \int_{-1}^{1} P(\rho) d \rho .
$$

We may again assume $s=0$. By Lemma 12 , for any $a>0$, the expression on the left may be written as the sum of the terms 


$$
\begin{aligned}
& \int_{-1}^{1} d \rho P(\rho)\left(1+\frac{\rho}{R}\right)^{\gamma} \cdot(R+\rho) \int_{0}^{a} f_{x}(t) H_{\alpha}(t(R+\rho)) d t \\
& \int_{a}^{\infty} d t f_{x}(t) \int_{-1}^{1} P(\rho)\left(1+\frac{\rho}{R}\right)^{\gamma}(R+\rho) H_{\alpha}(t(R+\rho)) d \rho .
\end{aligned}
$$

The argument leading up to Theorem III shows that under the condition (61), for every $a>0$,

$$
\lim _{R \rightarrow \infty} R \int_{0}^{a} f_{x}(t) H_{\alpha}(t R) d t=0 .
$$

Hence (143) goes to 0 if $R \rightarrow \infty$; and we have still to prove that (144) will be sufficiently small, uniformly in $R \geqq 2$, if only $a$ is sufficiently large. The latter will obviously be the case if the inner integral in (144) is $O\left(t^{-2}\right)$ for $t \rightarrow \infty$, uniformly in $R \geqq 2$. But, making use of (134), we see that the inner integral in (144) is, uniformly in $R \geqq 2$,

$$
\begin{aligned}
A_{0} \int_{-1}^{1} P(\rho)\left(1+\frac{\rho}{R}\right)^{\gamma} & \frac{e^{i t(R+\rho)}}{t} d \rho \\
& +B_{0} \int_{-1}^{1} P(\rho)\left(1+\frac{\rho}{R}\right)^{\gamma} \frac{e^{-i t(R+\rho)}}{t} d \rho+O\left(t^{-2}\right) .
\end{aligned}
$$

But the relation

$$
\begin{aligned}
\int_{-1}^{1} P(\rho)\left(1+\frac{\rho}{R}\right)^{\gamma} e^{i t \rho} d \rho=\frac{1}{i t} & {\left[P(\rho)\left(1+\frac{\rho}{R}\right)^{\gamma} e^{i t \rho}\right]_{-1}^{1} } \\
& -\frac{1}{i t} \int_{-1}^{1} \frac{d}{d \rho}\left[P(\rho)\left(1+\frac{\rho}{R}\right)^{\gamma}\right] e^{i t \rho} d \rho
\end{aligned}
$$

shows that (146) is also $O\left(t^{-2}\right)$, uniformly in $R \geqq 2$, and the proof of the lemma is completed.

LEMma 14. Given a positive integer $g$, there exists a polynomial $P(\rho)$ $=A_{0}+A_{1} \rho+A_{2} \rho^{2}+\cdots+A_{\sigma} \rho^{\sigma}$ such that

$$
\begin{aligned}
& \int_{-1}^{1} P(\rho) d \rho=1, \\
& \int_{-1}^{1} P(\rho) \rho^{\lambda} d \rho=0, \quad \lambda=1,2, \cdots, g .
\end{aligned}
$$

In order to obtain such a polynomial we may apply E. Schmidt's process of orthogonalization to the functions $t, t^{2}, \cdots, t^{0}, 1$ (in this order) on the interval $(-1,1)$. 
Lemma 15. Assumptions: (i) $P(t)$ satisfies (148), (149) for some $g>\alpha$; (ii) $N_{1}, N_{2}, \cdots$ is a sequence of points in the k-dimensional space having no finite point of accumulation and $\nu_{p}$ denotes the distance of $N_{p}$ from the origin; (iii) the constants $a\left(N_{1}\right), a\left(N_{2}\right), \cdots$ are such that

$$
\sum_{R \leqq \nu_{p} \leqq R+1}\left|a\left(N_{p}\right)\right|=o\left(R^{\alpha}\right) \quad \text { as } R \rightarrow \infty ;
$$

(iv) $A(R)$ and $B(R)$ are defined by

$$
\begin{aligned}
& A(R)=\sum_{\nu_{p} \leqq R}\left(R^{2}-\nu_{p}^{2}\right)^{\alpha} a\left(N_{p}\right), \\
& B(R)=\int_{-1}^{1} A(R+\rho) P(\rho) d \rho .
\end{aligned}
$$

Conclusion:

$$
\lim _{R \rightarrow \infty} \frac{A(R)-B(R)}{R^{2 \alpha}}=0 .
$$

It follows from (iii) that

$$
\sum_{R-2 \leqq \nu_{p} \leqq R}\left(R^{2}-\nu_{p}^{2}\right)^{\alpha} a\left(N_{p}\right)=o\left(R^{\alpha}\right)\left[R^{2}-(R-2)^{2}\right]^{\alpha}=o\left(R^{2 \alpha}\right) .
$$

Hence

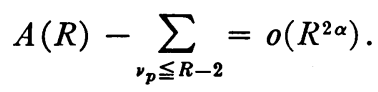

Substituting this in the right side of $B(R)$ we easily find

$$
B(R)-\sum_{\nu_{p} \leqq R-2} \int_{-1}^{1}\left((R+\rho)^{2}-\nu_{p}^{2}\right)^{\alpha} P(\rho) d \rho a\left(N_{p}\right)=o\left(R^{2 \alpha}\right) .
$$

Hence (152) will be proved if we show

$$
\sum_{\nu_{p} \leqq R-2} C\left(R, \nu_{p}\right) a\left(N_{p}\right)=o\left(R^{2 \alpha}\right),
$$

where

$$
C(R, \nu)=\left(R^{2}-\nu^{2}\right)^{\alpha}-\int_{-1}^{1}\left((R+\rho)^{2}-\nu^{2}\right)^{\alpha} P(\rho) d \rho
$$

Now

$$
\begin{aligned}
\left((R+\rho)^{2}-\nu^{2}\right)^{\alpha} & =\left(R^{2}-\nu^{2}+2 R \rho+\rho^{2}\right)^{\alpha} \\
& =\left(R^{2}-\nu^{2}\right)^{\alpha}\left(1+b_{1} \rho+\cdots+b_{g} \rho^{g}+b_{0+1}(\rho) \rho^{0+1}\right),
\end{aligned}
$$

where $b_{g+1}(\rho)$ uniformly in $\nu \leqq R-2$ and $|\rho| \leqq 1$, is majorized by 


$$
\left|\frac{2 R}{R^{2}-\nu^{2}}+\frac{1}{R^{2}-\nu^{2}}\right|^{0+1}
$$

which in its turn is majorized by

$$
\frac{1}{(R-\nu)^{g+1}} .
$$

Applying the decisive properties (148), (149) of $P(\rho)$ we find that, uniformly in $\nu \leqq R-2, C(R, \nu)$ is majorized by

$$
\frac{R^{\alpha}}{(R-\nu)^{g+1-\alpha}} .
$$

Moreover, for every fixed $\nu, C(R, \nu)=o\left(R^{2 \alpha}\right)$. Therefore, the left side of (156) is

$$
o(1) \cdot R^{\alpha} \int_{0}^{R-2} \frac{\nu^{\alpha} d \nu}{(R-\nu)^{\rho+1-\alpha}} .
$$

But for $g+1-\alpha>1$, that is, $g>\alpha$, the factor of $o(1)$ in (156) is $o\left(R^{2 \alpha}\right)$ and this proves relation (156).

THEOREM XIII. Let $f(x)$ be an almost periodic function of class $S$ whose Fourier exponents have no finite point of accumulation. If its Fourier coefficients are such that

$$
\sum_{R \leqq \nu_{p} \leqq R+1}\left|a_{n_{1} \cdots n_{k}}\right|=o\left(R^{(k-1) / 2}\right),
$$

then the (local) Lebesgue condition (61) is sufficient for the validity of (59).

The proof follows from Lemma 15, and Lemma 13 with $\gamma=2 \alpha$.

The number of lattice points in the $k$-dimensional space for which $R \leqq \nu_{p} \leqq R+1$ is $O\left(R^{k-1}\right)$ which, for $k \geqq 2$, is larger than $O\left(R^{(k-1) / 2}\right)$. Hence, for a periodic function of class $L$, (159) need not hold if $k \geqq 2$, and Theorem VII shows that (159) cannot be so relaxed as to be satisfied by any periodic function. But it follows from Bessel's inequality that (159) does hold if $f(x)$ belongs to the class $L_{2}$.

Corollary to TheOREM XIII. If $f(x)$ is a periodic function belonging to class $L_{2}$, then the (local) Lebesgue condition (61) is sufficient for the validity of (59).

It would be interesting to decide if this also holds for periodic functions belonging to class $L_{p}$, for $1<p<2$.

Princeton University,

Princeton, N. J. 